\title{
Effect of early tracheostomy on clinical outcomes in critically ill lung transplant recipients
}

\author{
Ryo Miyoshi ${ }^{1}$ (1) $\cdot$ Toyofumi F. Chen-Yoshikawa $^{1} \cdot$ Masatsugu Hamaji $^{1} \cdot$ Atsushi Kawaguchi $^{2} \cdot$ Hidenao Kayawake $^{1}$. \\ Kyoko Hijiya $^{1} \cdot$ Hideki Motoyama ${ }^{1} \cdot$ Akihiro Aoyama $^{1} \cdot$ Hiroshi Date $^{1}$
}

Received: 10 March 2018 / Accepted: 21 May 2018 / Published online: 23 May 2018

(c) The Japanese Association for Thoracic Surgery 2018

\begin{abstract}
Objectives The purpose of this study was to evaluate the effect of early tracheostomy in patients following lung transplantation and to determine its optimal timing and influence on clinical outcomes.

Methods We retrospectively reviewed records of 96 adult patients who underwent lung transplantation at our institution between August 2008 and January 2016. Time-to-tracheostomy was defined based on timing of the procedure: "early" if less than 3 days or "late" if 3 or more days after lung transplantation.

Results Forty-nine patients (51\%) underwent tracheostomy $3.2 \pm 1.8$ days after lung transplantation. Among these patients, 21 patients (42.9\%) underwent early tracheostomy and 28 patients (57.1\%) underwent late tracheostomy. Multivariable logistic regression analysis indicated that preoperative performance status was a significant predictor for tracheostomy $(p=0.006$, odds ratio 2.72). Patients in the early tracheostomy group began walking $(p=0.003)$ and oral feeding $(p=0.0006)$ earlier and had a shorter duration of mechanical ventilation $(p=0.04)$ and shorter length of intensive care unit $(p=0.01)$ and hospital stay $(p=0.04)$ than patients in the late tracheostomy group. No significant differences in postoperative walking $(p=0.06)$, oral feeding $(p=0.17)$, or length of hospital stay $(p=0.37)$ were observed between patients who underwent early tracheostomy and those who did not undergo tracheostomy.

Conclusions Early tracheostomy following lung transplantation decreased both intensive care and hospital stay, due to improved postoperative recovery, even in patients with poor preoperative conditions. Furthermore, length of hospital stay in patients with early tracheostomy was similar to that of patients without tracheostomy after lung transplantation.
\end{abstract}

Keywords Tracheostomy $\cdot$ Lung transplantation $\cdot$ Predictor $\cdot$ Hospital stay

\section{Introduction}

Lung transplantation is an established therapeutic option for patients with various end-stage lung diseases. Despite the advances in surgical techniques, use of immunosuppressants, and improvements in perioperative care, and organ preservation, patients undergoing lung transplantation often require prolonged mechanical ventilation.

Prolonged translaryngeal intubation is associated with an increased risk of complications, including

Hiroshi Date

hdate@kuhp.kyoto-u.ac.jp

1 Department of Thoracic Surgery, Kyoto University, 54 Shogoin-Kawahara-cho, Sakyo-ku, Kyoto 606-8507, Japan

2 Faculty of Medicine, Center for Comprehensive Community Medicine, Saga University, Saga, Japan ventilator-associated pneumonia and laryngeal injury [1]. Therefore, tracheostomy has been used to facilitate airway management and weaning in patients who require prolonged ventilatory assistance. Guide wire-assisted percutaneous tracheostomy techniques have been increasingly used in various patient populations, including critically ill patients [2], and surgical tracheostomy is performed only under specific circumstances.

There are potential benefits of early tracheostomy (ET) for critically ill patients. When compared with prolonged translaryngeal intubation or late tracheostomy (LT), ET can reduce the intensive care unit length of stay (LOS) and decrease duration of mechanical ventilation and hospital LOS [3-6]. However, there are little data assessing the benefit of ET in lung transplant patients despite the frequent use of tracheostomy after lung transplantation [7-10]. In addition, perioperative predictors for tracheostomy in this 
population have not been elucidated [11]. In this study, we assessed the effect of ET on clinical outcomes in patients after lung transplantation. We also investigated predictors for tracheostomy after lung transplantation.

\section{Patients and methods}

\section{Subjects}

A total of 105 adult patients underwent lung transplantation at our institution between August 2008 and January 2016. Three patients who required extracorporeal membrane oxygenation support and died of sepsis and multi-organ dysfunction were excluded from the study. In addition, six patients who had undergone tracheostomy before transplantation were also excluded. For this study, we retrospectively analyzed the records of 96 lung transplant recipients, including 44 who underwent living donor lobar lung transplantation and 52 who underwent cadaveric lung transplantation (14 bilateral and 38 unilateral).

All patients were transferred to the intensive care unit with intubation after lung transplantation. Extubation was considered under the following circumstances: (1) stable vital signs with a ratio of arterial oxygen partial pressure/ fractional inspired oxygen $\left(\mathrm{PaO}_{2} / \mathrm{FiO}_{2}\right)$ of $\geq 200 \mathrm{mmHg}$;

(2) stable underlying disease and lack of complications;

(3) minimal artificial ventilation with a low level of pressure support (5-8 cm water); and (4) spontaneous respirations less than 35 per minute. Extubation was done when these criteria were met during a spontaneous breathing trial [12]. A tracheostomy was performed when prolonged artificial mechanical ventilation was anticipated.
Tracheostomy was either performed surgically under general anesthesia in the operating room or percutaneously in the intensive care unit by the bedside.

Surgical tracheostomy for lung transplant patients was performed between August 2008 and August 2011. After September 2011, percutaneous tracheostomy was performed on all adult lung transplant patients who required prolonged artificial mechanical ventilation, with the exception of one patient with severe coagulopathy due to extracorporeal membrane oxygenation support. Percutaneous tracheostomy (Neo Perc ${ }^{\mathrm{TM}}$; Covidien Japan, Tokyo, Japan) was performed with direct bronchoscopic guidance.

\section{Methods}

Perioperative variables, obtained from our institution's lung transplant database and medical records, were analyzed retrospectively. Patients who underwent tracheostomy were divided into two groups based on the timing of tracheostomy: ET (performed less than 3-day posttransplantation) and LT (performed 3- or more day posttransplantation). We calculated propensity scores and adjusted the multivariable model to minimize selection bias. We used the Eastern Cooperative Oncology Group Performance Status (ECOG PS) to assess the activity of the patients and the Hugh-Jones classification to evaluate dyspnea before transplantation (Tables 1,2) [13, 14]. This study was approved by the institutional review board of Kyoto University Hospital (R0648). Written informed consent was waived because of the study's retrospective nature.

Table 1 Eastern Cooperative Oncology Group performance status [13]

\begin{tabular}{|c|c|}
\hline Grade 0 & Fully active, able to carry on all pre-disease performance without restriction \\
\hline Grade 1 & $\begin{array}{l}\text { Restricted in physically strenuous activity but ambulatory and able to carry out work of a light or sedentary nature; e.g., light } \\
\text { house work, office work }\end{array}$ \\
\hline Grade 2 & Ambulatory and capable of all self-care but unable to carry out any work activities; up and about more than $50 \%$ of waking hours \\
\hline Grade 3 & Capable of only limited self-care; confined to bed or chair more than $50 \%$ of waking hours \\
\hline Grade 4 & Completely disabled; cannot carry on any self-care; totally confined to bed or chair \\
\hline Grade 5 & Dead \\
\hline
\end{tabular}

Table 2 Hugh-Jones classification [14]

Grade 1 The patient's breathing is as good as that of healthy people of the same sex, age, and build while at work, walking, or climbing stairs

Grade 2 The patient is able to walk with other healthy people of the same sex, age, and build on a level surface but is unable to keep up while climbing stairs

Grade 3 The patient is unable to walk like other healthy people on a level surface but is able to walk a mile or more at a slower speed

Grade 4 The patient is unable to walk more than 100 yards on a level surface without rest

Grade 5 The patient is breathless while talking or changing clothes and is usually unable to leave home because of breathlessness 


\section{Outcomes}

The primary endpoint was hospital LOS. The secondary endpoints were factors predicting tracheostomy, the onset of postoperative walking, the onset of postoperative oral feeding, the total duration of mechanical ventilation, and the intensive care unit LOS. The endpoints were defined prior to the onset of the study.

\section{Statistics}

Continuous data are presented as mean \pm standard deviation. Categorical data are presented as numbers and percentages. The cutoff point between ET and LT was determined based on the point with the maximum differences in hospital LOS between the groups. After defining the cutoff point between ET and LT, the Student's unpaired $t$ test, Mann-Whitney $U$ test, and Fisher's exact test were used to identify significant between-group differences. Propensity scores were estimated using binary logistic regression. Propensity scores included the following variables: ECOG PS; type of transplantation; $\mathrm{PaO}_{2} / \mathrm{FiO}_{2}$ immediately after transplantation; cardiopulmonary support; operation time; and the ischemic time. To assess the timing of tracheostomy, the primary comparison was between ET and LT. However, to identify predictors for tracheostomy, comparisons were made between tracheostomy and no tracheostomy. To determine the effect of ET, comparisons were made between ET and no tracheostomy. Factors that were potentially or significantly associated with the requirement for tracheostomy were selected for multivariable analysis. The multivariable model included the following six variables: ECOG PS; type of transplantation; $\mathrm{PaO}_{2} /$ $\mathrm{FiO}_{2}$ immediately after transplantation; cardiopulmonary support; operation time; and ischemic time. Data analysis was performed using JMP, version 12 (SAS Institute Inc., Cary, USA), and $p<0.05$ was considered to be statistically significant.

\section{Results}

The baseline characteristics of patients are described in Table 3. Tracheostomy was performed in $49(51 \%)$ patients. The mean timing of tracheostomy was $3.2 \pm 1.8$ days (range 1-9 days) after lung transplantation. When compared to patients who did not undergo tracheostomy, patients who underwent tracheostomy had significantly poorer preoperative performance status $(p=0.0006)$ and longer operation time ( $p=0.003)$. When compared to patients who did not undergo tracheostomy, cardiopulmonary support was required more frequently in patients who did undergo tracheostomy $(p=0.005)$. In patients requiring cardiopulmonary support, 43 patients $(68.3 \%)$ required extracorporeal membrane oxygenation support. Multivariable logistic regression analysis indicated that the preoperative performance status was an independent predictor for tracheostomy after lung transplantation (odds ratio, 2.72; 95\% confidence interval, 1.33-6.07) (Table 4).

Table 5 shows the baseline characteristics of patients who underwent tracheostomy. Twenty-one patients (42.9\%) underwent ET and 28 patients (57.1\%) underwent LT. Percutaneous tracheostomy was performed in 39 patients (79.6\%). The mean intubation time for the ET group was $1.6 \pm 0.5$ days (range 1-2) and $4.3 \pm 1.6$ days (range $3-9$ ) for the LT group $(p<0.0001)$. There were no significant differences in the timing of the tracheostomy between patients with cadaveric single lung transplantation and patients with double lung transplantation $(p=0.09)$. Indications for tracheostomy were respiratory failure anticipated to require prolonged mechanical ventilation $(n=6)$ and profuse airway secretions resulting from severe graft dysfunction and/or pneumonia $(n=43)$ [15]. There were no major complications of tracheostomy.

Table 6 shows the clinical outcomes in the ET and LT groups. Early tracheostomy was associated with significantly earlier postoperative walking $(p=0.003)$ and oral feeding $(p=0.0006)$; a shorter duration of mechanical ventilation $(p=0.04)$; earlier removal of the tracheostomy tube ( $p=0.03)$; shorter intensive care unit LOS $(p=0.01)$; and shorter hospital LOS $(p=0.04)$. However, there were no significant differences in time to postoperative walking $(p=0.06)$ and oral feeding $(p=0.17)$ or hospital LOS $(p=0.37)$ between patients who underwent ET and those who did not undergo tracheostomy (Figs. 1,2).

In the multivariable model using propensity scores for ET, the timing of tracheostomy was an independent factor for the onset of postoperative walking $(p=0.02)$ and oral feeding $(p=0.02)$, the duration of mechanical ventilation $(p=0.04)$, and the intensive care unit LOS $(p=0.046)$. In addition, a trend existed between the timing of tracheostomy and the hospital LOS $(p=0.11)$.

\section{Discussion}

When compared to late tracheostomy, early tracheostomy in lung transplant patients is associated with significantly earlier postoperative recovery, including earlier postoperative walking and oral feeding, shorter duration of mechanical ventilation, and shorter intensive care unit and hospital LOS. Furthermore, postoperative recovery and hospital LOS in patients who underwent early tracheostomy was similar to that of patients who did not undergo tracheostomy. Poor preoperative performance status was the independent predictor for requiring a tracheostomy after lung transplantation.

Tracheostomy has several potential advantages over endotracheal intubation. It improves pulmonary hygiene, 
Table 3 Preoperative and intraoperative characteristics

\begin{tabular}{|c|c|c|c|}
\hline & Tracheostomy $(n=49)$ & No tracheostomy $(n=47)$ & $p$ value \\
\hline Age (years) & $44.4 \pm 14.0$ & $45.3 \pm 11.5$ & 0.74 \\
\hline Female & $22(44.9 \%)$ & $18(38.3 \%)$ & 0.54 \\
\hline Body mass index $\left(\mathrm{kg} / \mathrm{m}^{2}\right)$ & $19.6 \pm 4.1$ & $18.8 \pm 2.7$ & 0.29 \\
\hline \multicolumn{4}{|l|}{ Original disease } \\
\hline Idiopathic interstitial pneumonia & $15(30.6 \%)$ & $21(44.7 \%)$ & \\
\hline Other fibrosis & $15(30.6 \%)$ & $13(27.7 \%)$ & \\
\hline Chronic obstructive pulmonary disease & $6(12.2 \%)$ & $3(6.4 \%)$ & \\
\hline Primary pulmonary hypertension & $4(8.2 \%)$ & 0 & \\
\hline Cystic fibrosis or bronchiectasis & $3(6.1 \%)$ & $3(6.4 \%)$ & \\
\hline Lymphangioleiomyomatosis & $2(4.1 \%)$ & $4(8.5 \%)$ & \\
\hline Others & $4(8.2 \%)$ & $3(6.4 \%)$ & \\
\hline Requiring steroids & $26(53 \%)$ & $21(44.7 \%)$ & 0.42 \\
\hline Oxygen dependent & $48(97.9 \%)$ & $46(97.9 \%)$ & 1 \\
\hline Hospitalized before transplantation & $16(32.7 \%)$ & $10(21.3 \%)$ & 0.25 \\
\hline Previous history of colonization & $8(16.3 \%)$ & $14(29.8 \%)$ & 0.15 \\
\hline Hugh-Jones classification & & & 0.76 \\
\hline 2 & & 2 & \\
\hline 3 & 2 & 4 & \\
\hline 4 & 33 & 26 & \\
\hline 5 & 14 & 15 & \\
\hline Arterial carbon dioxide partial pressure $(\mathrm{mmHg})$ & $51.4 \pm 16.4$ & $48.6 \pm 10.4$ & 0.33 \\
\hline ECOG PS & & & 0.0006 \\
\hline 1 & & 1 & \\
\hline 2 & 18 & 33 & \\
\hline 3 & 24 & 10 & \\
\hline 4 & 7 & 3 & \\
\hline \multicolumn{4}{|l|}{ Type of transplantation } \\
\hline Living donor lobar & $26(53.1 \%)$ & $18(38.3 \%)$ & 0.16 \\
\hline Cadaveric single lung transplantation & $14(28.6 \%)$ & $24(51.1 \%)$ & \\
\hline Cadaveric double lung transplantation & $9(18.3 \%)$ & $5(10.6 \%)$ & \\
\hline $\mathrm{PaO}_{2} / \mathrm{FIO}_{2}$ immediately after lung transplantation $(\mathrm{mmHg})$ & $378.2 \pm 141.0$ & $383.6 \pm 134.1$ & 0.85 \\
\hline Cardiopulmonary support & $39(79.6 \%)$ & $24(51.1 \%)$ & 0.005 \\
\hline Cardiopulmonary support time (min) & $240.3 \pm 69.9$ & $211.5 \pm 53.7$ & 0.09 \\
\hline Ischemic time (min) & $283.2 \pm 165.9$ & $311.32 \pm 146.3$ & 0.38 \\
\hline Operation time $(\mathrm{min})$ & $464.4 \pm 120.3$ & $391.6 \pm 115.6$ & 0.003 \\
\hline
\end{tabular}

ECOG the Eastern Cooperative Oncology Group, $\mathrm{PS}$ performance status, $\mathrm{PaO}_{2}$ arterial oxygen partial pressure, $\mathrm{FiO}_{2}$ fractional inspired oxygen

Table 4 Multivariable logistic regression analysis of variables associated with tracheostomy

\begin{tabular}{|c|c|c|c|}
\hline & Odds ratio & $95 \%$ confidence interval & $p$ value \\
\hline ECOG PS & 2.72 & $1.33-6.07$ & 0.006 \\
\hline Operation time & 1.004 & 0.999-1.009 & 0.10 \\
\hline Cardiopulmonary support & 2.52 & $0.66-10.21$ & 0.18 \\
\hline Living donor lobar lung transplantation & 0.54 & $0.02-12.14$ & 0.70 \\
\hline Ischemic time & 0.999 & $0.990-1.008$ & 0.85 \\
\hline $\begin{array}{l}\mathrm{PaO}_{2} / \mathrm{FIO}_{2} \text { immediately after lung transplan- } \\
\text { tation }(\mathrm{mmHg})\end{array}$ & 0.997 & $0.992-1.001$ & 0.14 \\
\hline
\end{tabular}

ECOG the Eastern Cooperative Oncology Group, $\mathrm{PS}$ performance status, $\mathrm{PaO}_{2}$ arterial oxygen partial pressure, $\mathrm{FiO}_{2}$ fractional inspired oxygen 
Table 5 Baseline characteristics of patients with tracheostomy
Table 6 Clinical outcomes of patients with tracheostomy

\begin{tabular}{|c|c|c|c|}
\hline & $\begin{array}{l}\text { Early tracheos- } \\
\text { tomy }(n=21)\end{array}$ & $\begin{array}{l}\text { Late tracheos- } \\
\text { tomy }(n=28)\end{array}$ & $p$ value \\
\hline Age (years) & $45.7 \pm 13.2$ & $43.5 \pm 14.8$ & 0.57 \\
\hline Female & $7(33.3 \%)$ & $15(53.6 \%)$ & 0.25 \\
\hline Body mass index $\left(\mathrm{kg} / \mathrm{m}^{2}\right)$ & $20.5 \pm 3.7$ & $18.9 \pm 4.4$ & 0.11 \\
\hline \multicolumn{4}{|l|}{ Original disease } \\
\hline Idiopathic interstitial pneumonia & $7(33.3 \%)$ & $8(28.6 \%)$ & \\
\hline Lung injury after hematopoietic stem cell transplantation & $3(14.3 \%)$ & $8(28.6 \%)$ & \\
\hline Chronic obstructive pulmonary disease & $2(9.5 \%)$ & $4(14.3 \%)$ & \\
\hline Primary pulmonary hypertension & $3(14.3 \%)$ & $2(7.1 \%)$ & \\
\hline Other fibrosis & $1(4.8 \%)$ & $3(10.7 \%)$ & \\
\hline Others & $5(23.8 \%)$ & $3(10.7 \%)$ & \\
\hline Requiring steroids & $10(47.6 \%)$ & $16(57.1 \%)$ & 0.57 \\
\hline Oxygen dependent & $21(100 \%)$ & $28(100 \%)$ & \\
\hline Hospitalized before transplantation & $7(33.3 \%)$ & $9(32.1 \%)$ & 1 \\
\hline Previous history of colonization & $20(95.2 \%)$ & $21(75 \%)$ & 0.12 \\
\hline \multicolumn{4}{|l|}{ Hugh-Jones classification } \\
\hline 3 & 2 & & 0.93 \\
\hline 4 & 12 & 21 & \\
\hline 5 & 7 & 7 & \\
\hline Arterial carbon dioxide partial pressure $(\mathrm{mmHg})$ & $48.7 \pm 17.7$ & $53.4 \pm 15.5$ & 0.25 \\
\hline \multicolumn{4}{|l|}{ ECOG PS } \\
\hline 2 & 8 & 10 & 0.89 \\
\hline 3 & 10 & 14 & \\
\hline 4 & 3 & 4 & \\
\hline \multicolumn{4}{|l|}{ Type of transplantation } \\
\hline Living donor lobar & $10(47.6 \%)$ & $16(57.1 \%)$ & 0.57 \\
\hline Cadaveric single lung transplantation & $9(42.9 \%)$ & $5(17.9 \%)$ & \\
\hline Cadaveric double lung transplantation & $2(9.5 \%)$ & $7(25 \%)$ & \\
\hline $\mathrm{PaO}_{2} / \mathrm{FIO}_{2}$ immediately after lung transplantation $(\mathrm{mmHg})$ & $362.6 \pm 147.2$ & $389.9 \pm 137.7$ & 0.51 \\
\hline Cardiopulmonary support & $14(66.7 \%)$ & $25(89.3 \%)$ & 0.08 \\
\hline Cardiopulmonary support time (min) & $243.9 \pm 54.8$ & $238.4 \pm 78.0$ & 0.82 \\
\hline Ischemic time (min) & $311.1 \pm 177.0$ & $262.4 \pm 156.9$ & 0.31 \\
\hline Operation time (min) & $427.0 \pm 105.5$ & $492.5 \pm 124.9$ & 0.06 \\
\hline Major bleeding after lung transplantation & 1 & 3 & 0.63 \\
\hline Acute renal insufficiency after lung transplantation & 0 & 1 & 1 \\
\hline Postoperative profound coagulopathy or venoarterial ECMO & 1 & 6 & 0.21 \\
\hline Days from lung transplantation to tracheostomy & $1.6 \pm 0.5$ & $4.3 \pm 1.6$ & $<0.0001$ \\
\hline
\end{tabular}

ECOG the Eastern Cooperative Oncology Group, $\mathrm{PS}$ performance status, $\mathrm{PaO}_{2}$ arterial oxygen partial pressure, $\mathrm{FiO}_{2}$ fractional inspired oxygen, $E C M O$ extracorporeal membrane oxygenation support

\begin{tabular}{lccc}
\hline & $\begin{array}{l}\text { Early tracheos- } \\
\text { tomy }(n=21)\end{array}$ & $\begin{array}{l}\text { Late tracheostomy } \\
(n=28)\end{array}$ & $p$ value \\
\hline Onset of postoperative walking (days) & $6.1 \pm 2.5$ & $9.0 \pm 4.9$ & 0.003 \\
Onset of postoperative oral feeding (days) & $5.8 \pm 3.1$ & $10.1 \pm 6.5$ & 0.0006 \\
Total duration of mechanical ventilation (days) & $12.5 \pm 7.0$ & $19.9 \pm 13.9$ & 0.04 \\
Timing of removal of the tracheostomy tube (days) ${ }^{\mathrm{a}}$ & $25.5 \pm 9.4$ & $36.8 \pm 20.9$ & 0.03 \\
Intensive care unit LOS (days) & $11.9 \pm 6.3$ & $15.3 \pm 5.8$ & 0.01 \\
Hospital LOS (days) & $75.3 \pm 22.0$ & $105.5 \pm 86.2$ & 0.04 \\
\hline
\end{tabular}

LOS length of stay

${ }^{\text {a }}$ Time from transplantation to removal of the tracheostomy tube 


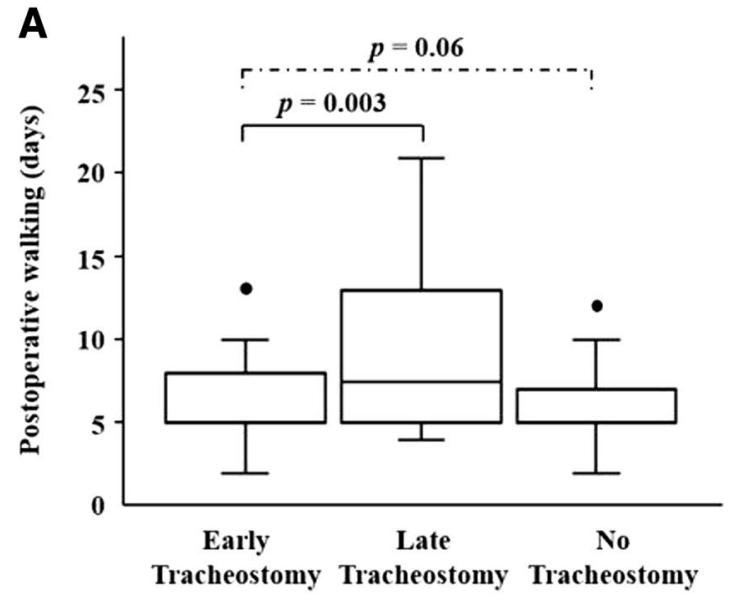

Fig. 1 Postoperative walking and oral feeding in the early tracheostomy, late tracheostomy, and no tracheostomy groups. a Patients in the early tracheostomy group began postoperative walking significantly earlier than those in the late tracheostomy group $(p=0.003)$. However, there were no significant differences between the early tra-

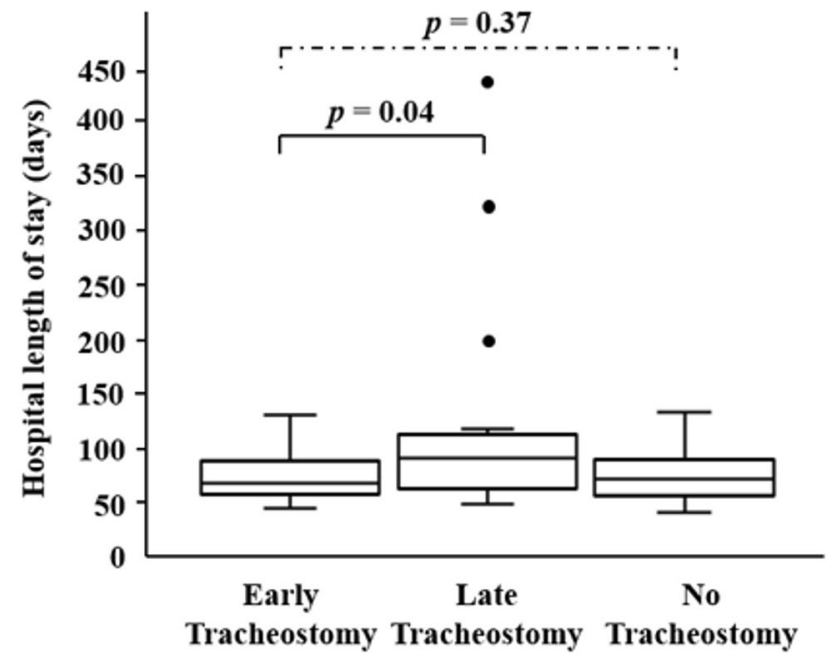

Fig. 2 Hospital length of stay in the early tracheostomy, late tracheostomy, and no tracheostomy groups. Early tracheostomy was associated with a significantly shorter hospital length of stay when compared to the late tracheostomy group $(p=0.04)$. There were no significant differences between the early tracheostomy and no tracheostomy groups $(p=0.37)$

facilitates weaning from mechanical ventilation, secures airway control, and helps achieve oral feeding and mobilization [16]. Although lung transplantation is an established therapeutic option for various end-stage lung diseases, mortality remains high. According to the 2016 registry of the International Society for Heart and Lung Transplantation, the 1-year post-transplant survival for patients undergoing lung transplantation was approximately $80 \%$ [17]. In the acute phase of lung transplantation, respiratory failure and

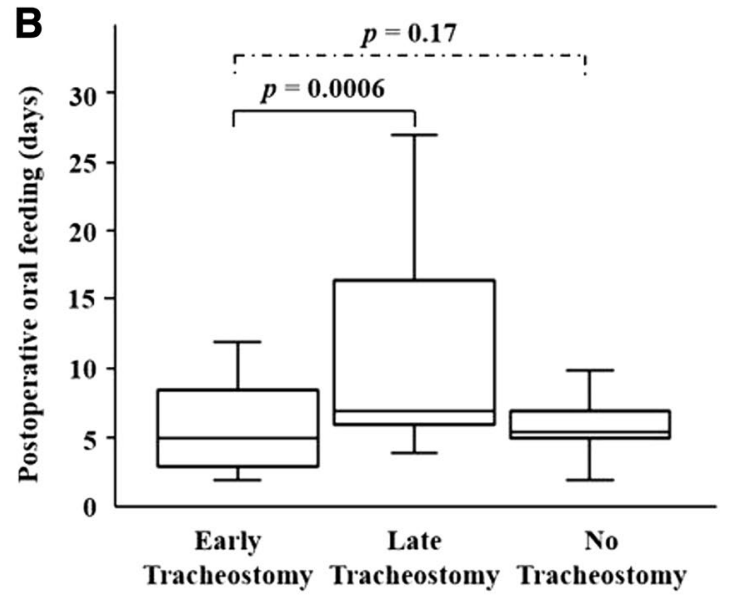

cheostomy and no tracheostomy groups $(p=0.06)$. b Patients in the early tracheostomy group began postoperative oral feeding significantly earlier than those in the late tracheostomy group $(p=0.0006)$. However, there were no significant differences between the early tracheostomy and no tracheostomy groups $(p=0.17)$

infection are considered to be the major causes of death [17]. Due to respiratory muscle fatigue and ineffective pulmonary function, lung transplant patients are likely to develop postoperative acute respiratory failure, and may require prolonged mechanical ventilation. In the general intensive care unit population, ET is useful for patients requiring prolonged mechanical ventilation [4-6]. However, in the lung transplant population, there are no reports investigating the relationship between the timing of tracheostomy and clinical outcomes. Therefore, we conducted the present study to assess the effect of ET on clinical outcomes after lung transplantation.

Early postoperative recovery, such as postoperative walking and oral feeding, are considered important to shorten the hospital LOS and improve patient outcomes [18, 19]. We found that ET was associated with significantly earlier postoperative walking and oral feeding. Moreover, ET was associated with a significantly shorter duration of mechanical ventilation, earlier removal of the tracheostomy tube, and a shorter intensive care unit and hospital LOS. These findings suggest that early postoperative recovery, including postoperative walking, oral feeding, and removal of the tracheostomy tube, may reduce hospital LOS in patients with ET. Multivariable analysis with propensity scores for ET showed that the timing of tracheostomy was significantly associated with the onset of postoperative walking and oral feeding; the duration of mechanical ventilation; and the intensive care unit LOS. The timing of the tracheostomy was not significantly associated with hospital LOS; however, the lack of significance may be attributed to unknown confounders and the relatively small sample size. While the impact of ET in different populations has been examined [4-6], the 
definition of the ET based on timing varies. Despite the variations in definitions, ET reduced the duration of mechanical ventilation and intensive care unit LOS and/or hospital LOS. Although there were no lung transplant patients in these studies, the findings from these studies are in agreement with our findings. Moreover, we found that postoperative recovery and hospital LOS in patients who underwent ET were similar to patients who did not undergo tracheostomy. Although patients undergoing tracheostomy had poorer general condition than those who did not undergo tracheostomy, there was a significant benefit from ET.

We identified important potential predictors for tracheostomy after lung transplantation. Intraoperative factors included prolonged operation time and the use of a cardiopulmonary support system. In agreement with the results from our study, Pedia et al. reported that cardiopulmonary bypass is one of the predictors of tracheostomy [8]. In terms of preoperative factors, poor preoperative performance status was an independent predictor of tracheostomy. Poor performance status may be due to reduced muscle mass and muscle weakness, as a consequence of severe respiratory failure. Patients with poor performance status may have poor nutritional status and systemic muscle weakness that may be associated with postoperative respiratory muscle fatigue and poor clearance of airway secretions. This may predispose lung transplant patients to tracheostomy.

Percutaneous tracheostomy offers several advantages over surgical tracheostomy and has become the preferred choice for lung transplant patients $[9,10,20]$. In a meta-analysis comparing surgical versus percutaneous tracheostomy, Freeman et al. showed that the percutaneous method reduced operation time, perioperative bleeding, wound infection, and postoperative complications [20]. Generally, in the acute phase, complications associated with tracheostomy include bleeding, tracheal injury, cannula dislocation, hypoxia, pneumothorax, and infection. Late complications include tracheal stenosis. In agreement with results from previous studies $[9,10]$, in our study, percutaneous tracheostomy was performed in 39 patients without major complications. However, the proportion of patients who underwent tracheostomy in our study is greater than previously reported $[9,10]$. This may reflect the severe donor shortage in Japan [21]. For critically ill patients in Japan, transplantation of marginal lungs from brain dead donors or living donor transplantation for patients considered too critically ill to wait for brain dead donor lungs, may be done. The difference between lung graft conditions and the general preoperative condition of patients in Japan, when compared to that in other countries, may explain the increased use of tracheostomy and the relative long hospital LOS in this study.

There are several limitations to our study. First, it is a retrospective, single institutional study. Because the database was not designed specifically to examine tracheostomy, certain important factors were not investigated, including operation time of tracheostomy or the trend in the $\mathrm{PaO}_{2} /$ $\mathrm{FiO}_{2}$ and the acute physiology and chronic health evaluation (APACHE) score after transplantation. Although a tracheostomy was performed when prolonged artificial mechanical ventilation was anticipated, there are no criteria for tracheostomy. This subjective consideration of performing tracheostomy is also an important limitation. We could not precisely assess the relationship between the change in the patient condition and the timing of the tracheostomy, because we did not record the trend in the $\mathrm{PaO}_{2} / \mathrm{FiO}_{2}$ and the APACHE score after transplantation. The ET timing defined in this study was shorter than that in the previous studies [4-6, 22]. The severe donor shortage in Japan may have resulted in a greater proportion of patients in our study undergoing tracheostomy when compared to lung transplant patients in other countries [9, 10,21]. Approximately half of the patients in our study underwent living donor lobar lung transplantation and the proportion of patients with intraoperative cardiopulmonary support was relatively high. These unique backgrounds with the severe donor shortage in Japan may contribute to the relative long hospital LOS in this study in comparison to that in other countries. To assess the preoperative general condition and respiratory status of patients, we used the ECOG PS and the Hugh-Jones classification instead of the lung allocation score, because it is not used in Japan $[11,13,14]$. We did not distinguish primary graft dysfunction from pneumonia, because bacterial infection may present a clinical profile similar to graft dysfunction, and may accompany graft dysfunction [15]. Because the lung grafts are deemed to be susceptible to primary graft dysfunction within $72 \mathrm{~h}$ following lung transplantation, early tracheostomy might contribute to worsening primary graft dysfunction. Furthermore, the number of patients included in this study was small and our results may have been influenced by selection bias, because we did not adjust for the unknown characteristics of the patients. Therefore, a larger, prospective, multi-institutional, and international study is warranted to confirm our findings.

\section{Conclusion}

Early tracheostomy significantly reduced intensive care unit and hospital LOS due to improved postoperative recovery after lung transplantation. Postoperative recovery and hospital LOS in patients who underwent early tracheostomy were similar to those in patients who did not undergo tracheostomy. Poor preoperative performance status was the independent predictor for tracheostomy after lung transplantation. Thus, in the unique backgrounds with the severe donor shortage in Japan, our study suggests that early tracheostomy may improve post-lung transplant clinical outcomes. 
Acknowledgements We would like to thank Dr. Keisuke Hamamura from the Department of Gastroenterology and Hepatology, Shizuoka City Shizuoka Hospital for help in the methodological discussions.

\section{Compliance with ethical standards}

Conflict of interest The authors have declared that no conflict of interest exists.

\section{References}

1. Santos PM, Afrassiabi A, Weymuller EA. Risk factors associated with prolonged intubation and laryngeal injury. Otolaryngol Head Neck Surg. 1994;111:453-9.

2. Ciaglia P, Firsching R, Syniec C. Elective percutaneous dilatational tracheostomy. A new simple bedside procedure; preliminary report. Chest. 1985;87(6):715-9.

3. Kollef MH, Ahrens TS, Shannon W. Clinical predictors and outcomes for patients requiring tracheostomy in the intensive care unit. Crit Care Med. 1999;27:1714-20.

4. Armstrong PA, McCarthy MC, Peoples JB. Reduced use of resources by early tracheostomy in ventilator-dependent patients with blunt trauma. Surgery. 1998;124:763-6.

5. Arabi Y, Haddad S, Shirawi N, Al Shimemeri A. Early tracheostomy in intensive care trauma patients improves resource utilization: a cohort study and literature review. Crit Care. 2004;8:R347-R52.

6. Koch T, Hecker B, Hecker A, Brenck F, Preuß M, Schmelzer T, et al. Early tracheostomy decreases ventilation time but has no impact on mortality of intensive care patients: a randomized study. Langenbecks Arch Surg. 2012;397(6):1001-8.

7. Chen F, Chin K, Sato M, Aoyama A, Murase K, Azuma M, et al. Postoperative respiratory management in living donor lobar lung transplantation. Clin Transplant. 2013;27(4):E383-90.

8. Padia SA, Borja MC, Orens JB, Yang SC, Jhaveri RM, Conte JV. Tracheostomy following lung transplantation predictors and outcomes. Am J Transplant. 2003;3(7):891-5.

9. Feltracco P, Milevoj M, Alberti V, Carollo C, Michieletto E, Rea $\mathrm{F}$, et al. Early tracheostomy following lung transplantation. Transplant Proc. 2011;43:1151.

10. Pilarczyk K, Carstens H, Heckmann J, Lubarski J, Marggraf G, Jakob H, et al. Safety and efficiency of percutaneous dilatational tracheostomy with direct bronchoscopic guidance for thoracic transplant recipients. Respir Care. 2016;61(2):235-42.

11. Russo MJ, Davies RR, Hong KN, Iribarne A, Kawut S, Bacchetta $\mathrm{M}$, et al. Who is the high-risk recipient? Predicting mortality after lung transplantation using pretransplant risk factors. J Thorac Cardiovasc Surg. 2009;138(5):1234-8.

12. MacIntyre NR, Cook DJ, Ely EW, Epstein SK, Fink JB, Heffner $\mathrm{JE}$, et al. Evidence-based guidelines for weaning and discontinuing ventilatory support: a collective task force facilitated by the American College of Chest Physicians; the American Association for Respiratory Care; and the American College of Critical Care Medicine. Chest. 2001;120(6 Suppl):375S-95S.

13. Oken MM, Creech RH, Tormey DC, Horton J, Davis TE, McFadden ET, et al. Toxicity and response criteria of the Eastern Cooperative Oncology Group. Am J Clin Oncol. 1982;5(6):649-55.

14. Hugh-Jones P, Lambert AV. A simple standard exercise test and its use for measuring exertion dyspnea. Br Med J 1952;1(4749):65-71.

15. Christie JD, Carby M, Bag R, Corris P, Hertz M, Weill D, et al. Report of the ISHLT Working Group on Primary Lung Graft Dysfunction part II: definition. A consensus statement of the International Society for Heart and Lung Transplantation. J Heart Lung Transplant. 2005;24(10):1454-9.

16. Pryor JP, Reilly PM, Shapiro MB. Surgical airway management in the intensive care unit. Crit Care Clin. 2000;16(3):473-88.

17. Yusen RD, Edwards LB, Dipchand AI, Goldfarb SB, Kucheryavaya AY, Levvey BJ, et al. The Registry of the International Society for Heart and Lung Transplantation: thirty-third official adult lung and heart-lung transplantation report-2016; focus theme: primary diagnostic indications for transplant. J Heart Lung Transplant. 2016;35(10):1170-84.

18. Hur H, Kim SG, Shim JH, Song KY, Kim W, Park CH, et al. Effect of early oral feeding after gastric cancer surgery: a result of randomized clinical trial. Surgery 2011;149(4):561-8.

19. Das-Neves-Pereira JC, Bagan P, Coimbra-Israel AP, GrimaillofJunior A, Cesar-Lopez G, Milanez-de-Campos JR, et al. Fast-track rehabilitation for lung cancer lobectomy: a five-year experience. Eur J Cardiothorac Surg. 2009;36(2):383-91. (discussion 391-2).

20. Freeman BD, Isabella K, Lin N, Buchman TG. A meta-analysis of prospective trials comparing percutaneous and surgical tracheostomy in critically ill patients. Chest. 2000;118:1412-8.

21. Miyoshi R, Chen-Yoshikawa TF, Hijiya K, Motoyama H, Aoyama A, Menju T, et al. Significance of single lung transplantation in the current situation of severe donor shortage in Japan. Gen Thorac Cardiovasc Surg. 2016;64(2):93-7.

22. Young D, Harrison DA, Cuthbertson BH, Rowan K, TracMan C. Effect of early vs late tracheostomy placement on survival in patients receiving mechanical ventilation: the TracMan randomized trial. JAMA. 2013;309(20):2121-9. 